\title{
On the electro-sensing of weakly electric fish
}

\author{
Eric Bonnetier ${ }^{\dagger}$, Faouzi Triki ${ }^{\ddagger}$, and Chun-Hsiang Tsou ${ }^{\S}$
}

\begin{abstract}
In this paper we are interested in electro-sensing inverse problems. Our objective is to understand the electro-perception mechanism of weakly electric fish. These species of fish have the ability to recognize the environment around them in complete darkness by generating a weak electrical field at different frequencies, and perceiving the transdermal potential perturbation. Assuming that the target has a known conductivity profile, the electro-sensing inverse problem consists in recovering the shape and location of the target from measurements of the electric potential over the skin. Using an original spectral decomposition of the solution to the direct problem in terms of Poincaré variational eigenfunctions, we retrieve the Cauchy data of the electric potential over the fish skin corresponding to the case where we substitute the target by a perfect conductor with the same shape and position. We then identify the target from the recovered Cauchy data. We derive uniqueness and stability estimates to the considered electro-sensing inverse problem. The numerical validation of our theoretical approach is realized by reconstructing different targets using synthetic data in dimension two. The numerical experiments are conducted using gradient descent optimization algorithms.
\end{abstract}

\section{Contents}

1. Introduction

2. The Mathematical Model

3. Well-posedness of the direct problem 4

4. The Poincaré Variational Problem 8

5. Spectral decomposition of the solution $u(x, \omega) \quad 11$

6. Uniqueness and stability estimates 13

7. Numerical identification of the target fish 14

8. Conclusion 20

9. Acknowledgments 20

References $\quad 21$

1991 Mathematics Subject Classification. Primary: 35R30.

Key words and phrases. Inverse problems, uniqueness, multifrequency, unique continuation, electrolocalization, stability estimates, weakly electric fish. 


\section{Introduction}

Some species of fish have the ability to recognize the environment around them by generating a weak electrical field at different frequencies. Their sensorial organs can generate electric fields, and their skins are sensitive to nearby electric fields. Their central nervous systems can identify objects by the response perceived on their skin to the electric impulses they generated. From the mathematical point of view these electric waves can be described by the Maxwell equations in the quasi-statistic regime, and understanding the behavior of the electrical fields in the neighborhood of the fish leads to an inverse conductivity problem with a finite number of excitations at different frequencies, and a finite number of boundary measurements.

To identify its targets the weak electric fish solves an inverse problem that has many similarities with the multifrequency electric impedance tomography. This latter method is a recent imaging technique of biological tissues where one tries to take advantage of the fact that most biological tissues exhibit frequency dependent conductivities, when excited by electric waves with frequencies ranging roughly around $10 \mathrm{kHz}[\mathbf{9}, \mathbf{6}, \mathbf{1 8}]$. Experiments indicate that electric fish produce time harmonic electric waves at different frequencies, to gather information about their environment. Assuming that the target is a different fish, our objective here is to study how a weakly electric fish might identify it. The proposed inverse problem has many potential bio-inspired applications in underwater robotics $[\mathbf{1 6}, \mathbf{1 4}]$.

In this paper we are interested in the case where the electric fish and the target fish occupy respectively the bounded domains $\Omega \subset \mathbb{R}^{d}, d=2,3$, and $D \subset \mathbb{R}^{d} \backslash \bar{\Omega}$. We assume that the conductivity distribution around the electric fish, is given by

$$
\gamma(x, \omega)=1+(k(\omega)-1) \chi_{D}(x),
$$

where $\chi_{D}$ denotes the characteristic function of $D$. The conductivity of the background is normalized to be equal to one, $k(\omega): \mathbb{R}_{+} \rightarrow \mathbb{C} \backslash \overline{\mathbb{R}_{-}}$denotes the conductivity of the target fish, and $\omega$ the frequency of the electric wave $u$ produced by the electric fish. Recently, shape recognition and classification methods have been applied on small volume target fish $[\mathbf{4}, \mathbf{5}]$. In the present work we adapt the method developed in $[\mathbf{9}]$ to the weak electric fish inverse problems. We take advantage of multifrequency measurements and combine unique continuation techniques for meromorphic complex functions with a particular spectral representation of $u$ that involves the eigenfunctions of the Poincaré variational operator, to reconstruct the conductivity map $\gamma$. The spectral decomposition can be written in the form

$$
u(x, \omega)=u_{0}(x)+u_{f}(x, k(\omega)),
$$

where the function $u_{0}$ is independent of the frequency $\omega$, and represents the limiting solution when the contrast $k(\omega)$ tends to $\infty$. In fact $u_{0}$ is exactly the electric potential when we substitute the target fish by a perfect conductor with the same shape and position. Thus, it is not surprising that one can uniquely identify $D$ from only one boundary measurement $\left.u_{0}\right|_{\partial \Omega}$. The problem of determining $D$ from $\left.u_{0}\right|_{\partial \Omega}$ has been studied by several authors (see e.g. $[\mathbf{1 3}, \mathbf{2}]$ ).

In this paper, we are concerned with the multifrequency inverse inclusion problem in unbounded domains. We first describe the mathematical model of electroreception. After introducing the weighted Sobolev space $\mathcal{W}^{1,-1}\left(\mathbb{R}^{d}\right)$, which is the natural space for solving the Laplace equation using variational techniques in 
unbounded domains, we prove the existence and uniqueness of solutions to the direct problem. We then set up a spectral decomposition using a modified Poincaré variational operator on $\mathcal{W}^{1,-1}\left(\mathbb{R}^{d}\right)$. Using the same techniques of reconstruction as in $[\mathbf{9}]$, we derive the uniqueness of solutions to the inverse problem, and obtain stability estimates. To validate our theoretical approach we reconstruct different targets using synthetic data in dimension two.

This paper is organized as follows. In the next section, we present the mathematical model of the weakly electric fish. In section 3, we study the well-posedness of the partial differential system associated to the forward problem. In section 4, we introduce the Poincaré variational operator, and study its spectrum. Next, we derive the spectral decomposition of the unique solution to the forward problem in section 5. We derive uniqueness and stability estimates to the inverse problem in section 6 . Section 7 is devoted to numerical illustrations of our theoretical results. A similar spectral decomposition is used to derive the frequency independent part $u_{0}$ in a bounded truncated numerical domain. We introduce an optimization scheme to reconstruct the position and shape of the target fish and present some numerical experiments.

\section{The Mathematical Model}

In this section we adapt the mathematical model of the weakly electric fish introduced by Ammari and al. in [3]. Let $\Omega \subset \mathbb{R}^{d}, d=2,3$, be an open connected and bounded region, which represents the electric fish. We suppose that $\partial \Omega$ is of class $\mathcal{C}^{1, \alpha}$ for some $\alpha \in(0,1]$. We denote by $\Omega_{e}$ the exterior of $\Omega$, that is $\Omega_{e}:=\mathbb{R}^{d} \backslash \bar{\Omega}$.

The target fish $D$ is also assumed to be an open connected region in $\Omega_{e}$. We assume that there exists $\delta>0$ such that $\operatorname{dist}(\partial \Omega, \partial D)>\delta$. We define a class of inclusions on which we study the uniqueness and stability of the inverse problem. We assume that the inclusion $D$ contains the origin. Let $b_{1}=\operatorname{dist}(0, \partial \Omega)$ and let $b_{0}<b_{1}$. For $m>2$ and $\varsigma \in(0,1]$, we define the class of inclusions

$$
\mathfrak{D}:=\left\{D:=\left\{x \in \mathbb{R}^{d}:|x|<\Upsilon(\hat{x}), \hat{x}=\frac{x}{|x|}\right\} ; b_{0}<\Upsilon(\hat{x})<b_{1}-\delta ;\|\Upsilon\|_{\mathcal{C}^{2, \varsigma}} \leq m\right\} .
$$

We assume that the conductivity is equal to 1 everywhere in $\Omega_{e}$ except in the target $D$ where the conductivity is equal to $k(\omega)$. We denote by $\gamma(x, \omega):=$ $1+(k(\omega)-1) \chi_{D}$, the conductivity distribution in $\Omega_{e}$. Let $u$ be the electric voltage produced by the electric fish, which satisfies the following system

$$
\left\{\begin{array}{lr}
\triangle u=J_{s} & \text { in } \Omega, \\
\operatorname{div}[\gamma(x, \omega) \nabla u]=0 & \text { in } \Omega_{e}, \\
\left.\partial_{\nu} u\right|_{-}=0 & \text { on } \partial \Omega, \\
\left.u\right|_{+}-\left.u\right|_{-}=\left.\xi \partial_{\nu} u\right|_{+} & \text {on } \partial \Omega, \\
|u|=O\left(\frac{1}{|x|^{d-1}}\right) & \text { as }|x| \rightarrow \infty,
\end{array}\right.
$$

where the constant $\xi>0$ is the effective fish skin thickness, where $J_{s}$ represents the electric current source generated by the fish organs, and where $\partial_{\nu}$ denotes the derivative with respect to the outward normal vector $\nu$. We assume that $J_{s}$ can be written as the sum of Dirac masses

$$
J_{s}=\sum_{j=1}^{M} \alpha_{j} \delta_{x_{s}^{(j)}},
$$


where $x_{s}^{(j)} \in \Omega$ and the electric charges $\alpha_{j} \in \mathbb{R}$ satisfy the neutrality condition

$$
\sum_{j=1}^{M} \alpha_{j}=0
$$

Note that the equation (2.1) can also be rewritten as

$$
\left\{\begin{array}{lr}
\triangle u=J_{s} & \text { in } \Omega, \\
\triangle u=0 & \text { in } \Omega_{e} \backslash \partial D, \\
\left.\partial_{\nu} u\right|_{-}=0 & \text { on } \partial \Omega, \\
\left.u\right|_{+}-\left.u\right|_{-}=\left.\xi \partial_{\nu} u\right|_{+} & \text {on } \partial \Omega, \\
\left.u\right|_{+}=\left.u\right|_{-} & \text {on } \partial D \\
\left.\partial_{\nu} u\right|_{+}=\left.k(\omega) \partial_{\nu} u\right|_{-} & \text {on } \partial D, \\
|u|=O\left(\frac{1}{|x|^{d-1}}\right) & \text { as }|x| \rightarrow \infty .
\end{array}\right.
$$

Assuming that $k(\omega)$ is a given continuous function, the inverse problem of weakly electric fish consists in recovering the shape and the position of the inclusion $D$ from measurements of the voltages $u(x, \omega)$ on the boundary $\partial \Omega$ for $\omega \in(\underline{\omega}, \bar{\omega})$, where $\underline{\omega}, \bar{\omega}$ are fixed constants.

\section{Well-posedness of the direct problem}

In this section, we study the well-posedness of the direct problem (2.1). Firstly, we introduce the Sobolev space $\mathcal{W}^{1,-1}\left(\Omega_{e}\right)$. Secondly, we establish the existence and uniqueness of the solution to $(2.1)$ in $\mathcal{W}^{1,-1}\left(\Omega_{e}\right)$.

3.1. Sobolev space $\mathcal{W}^{1,-1}\left(\Omega_{e}\right)$. In this subsection, we establish using variational techniques the existence and uniqueness for the Laplace equations with a Robin boundary condition in $\Omega_{e}$. To overcome the difficulties of integrating by parts in the unbounded exterior domain $\Omega_{e}$, we introduce the following weighted Sobolev spaces $[\mathbf{1 9}]$.

\section{DeFinition 3.1.}

$$
\mathcal{W}^{1,-1}\left(\Omega_{e}\right):=\left\{u ; \frac{u(x)}{\left(1+|x|^{2}\right)^{1 / 2}\left(\log \left(2+|x|^{2}\right)\right)^{3-d}} \in L^{2}\left(\Omega_{e}\right), \nabla u \in L^{2}\left(\Omega_{e}\right)\right\} .
$$

REMARK 3.1. We make the following observations.

- From the boundedness of the weighted functions, $\mathcal{W}^{1,-1}(U)$ is identically equal to the usual Sobolev space $H^{1}(U)$ on any open bounded domain $U$.

- The space of infinitely differentiable functions with compact support $\mathcal{D}\left(\Omega_{e}\right)$ is dense in the subspace $\mathcal{W}_{0}^{1,-1}\left(\Omega_{e}\right):=\left\{u \in \mathcal{W}^{1,-1}\left(\Omega_{e}\right) ;\left.u\right|_{\partial \Omega}=0\right\}$.

- The functions $v \in \mathcal{W}^{1,-1}\left(\Omega_{e}\right)$ satisfy the following decay behavior far from $\Omega$

$$
v(x)=O\left(\frac{1}{|x|^{d-2}}\right), \quad|x| \rightarrow+\infty .
$$

In particular, the constant functions belong to $\mathcal{W}^{1,-1}\left(\Omega_{e}\right)$ when $d=2$. 
We introduce the space

$$
\mathcal{L}_{-1}^{2}\left(\Omega_{e}\right):=\left\{u ; \frac{u(x)}{\left(1+|x|^{2}\right)^{1 / 2}\left(\log \left(2+|x|^{2}\right)\right)^{3-d}} \in L^{2}\left(\Omega_{e}\right)\right\} .
$$

We state now a useful compact embedding result involving these weighted Sobolev spaces.

Lemma 3.1. The embedding $\mathcal{W}^{1,-1}\left(\Omega_{e}\right) \rightarrow \mathcal{L}_{-1}^{2}\left(\Omega_{e}\right)$ is compact.

The proof follows the same approach used in $[\mathbf{1}]$.

Proof. If the embedding $\mathcal{W}^{1,-1}\left(\mathbb{R}^{d}\right) \rightarrow \mathcal{L}_{-1}^{2}\left(\mathbb{R}^{d}\right)$ is compact, then it is also compact for any connected domain $O \subset \mathbb{R}^{d}$. So, without loss of generality, we only consider the case $\mathbb{R}^{d}, d=2$.

Let $u \in \mathcal{D}\left(\mathbb{R}^{2}\right)$, we have

$$
u(\rho, \theta)=-\int_{\rho}^{\infty} \partial_{r} u(r, \theta) d r
$$

where $(r, \theta)$ are the polar coordinates.

Then,

$$
\frac{|u(\rho, \theta)|^{2}}{\left(1+\rho^{2}\right)\left(\log \left(2+\rho^{2}\right)^{2}\right)} \leq C \frac{1}{\rho^{2}(\log (\rho))^{2}} \int_{\rho}^{\infty}\left|\partial_{r} u(r, \theta) u(r, \theta)\right| d r,
$$

for $\rho \geq \rho_{0}>0$.

Denoting by $B_{R}^{c}$ the exterior of the ball $B_{R}=B(0, R)$, we have,

$$
\begin{aligned}
& \int_{B_{R}^{c}} \frac{|u(x)|^{2}}{\left(1+|x|^{2}\right) \log \left(2+|x|^{2}\right)^{2}} d x \\
& \leq C \int_{0}^{2 \pi} d \theta \int_{R}^{\infty} \frac{1}{\rho(\log (\rho))^{2}} \int_{\rho}^{\infty}\left|\partial_{r} u(r, \theta) u(r, \theta)\right| d r d \rho \\
& \leq C \frac{1}{\log (R)}\|u\|_{\mathcal{W}^{1,-1}\left(\mathbb{R}^{2}\right)}^{2}
\end{aligned}
$$

By density, this inequality holds for any $u \in \mathcal{W}^{1,-1}\left(\mathbb{R}^{2}\right)$. Let $\left(u_{i}\right)_{i \in \mathbb{N}}$ be a bounded sequence in $\mathcal{W}^{1,-1}\left(\mathbb{R}^{2}\right)$. To prove that this sequence is a precompact in $\mathcal{L}_{-1}^{2}\left(\mathbb{R}^{2}\right)$, it is sufficient to show

i) for $\varepsilon>0$, there exists $R$ such that $\left\|u_{i}\right\|_{\mathcal{L}_{-1}^{2}\left(B_{R}^{c}\right)}<\varepsilon$ for all $i$.

ii) for any bounded part $\mathcal{O} \subset \mathbb{R}^{2},\left(\left.u_{i}\right|_{\mathcal{O}}\right)_{i \in \mathbb{N}}$ is a precompact.

The first point is a direct consequence of the previous inequality. The second point can be obtained by applying the Rellich-Kondrachov Theorem. Thus, the result of the lemma follows.

3.2. Well-posedness. In this subsection we establish the existence and uniqueness to $(2.1)$ in $\mathcal{W}^{1,-1}\left(\Omega_{e}\right)$.

Lemma 3.2. If $d=2$, let $\alpha>0$. Then there exists $\beta>0$ such that $\forall u \in$ $\mathcal{W}^{1,-1}\left(\Omega_{e}\right)$,

$$
\int_{\Omega_{e}}|\nabla u|^{2} d x+\alpha \int_{\partial \Omega} u^{2} d \sigma \geq \beta\|u\|_{\mathcal{L}_{-1}^{2}\left(\Omega_{e}\right)}^{2}
$$


Proof. We suppose by a contradiction argument that (3.6) is not true. The opposite of the statement of the lemma implies that there exists a sequence $\left(u_{m}\right)_{m \in \mathbb{N}}$ with $u_{m} \in \mathcal{W}^{1,-1}\left(\Omega_{e}\right),\left\|u_{m}\right\|_{\mathcal{W}^{1,-1}\left(\Omega_{e}\right)}=1$, such that

$$
\int_{\Omega_{e}}\left|\nabla u_{m}\right|^{2} d x+\alpha \int_{\partial \Omega} u_{m}^{2} d \sigma<\frac{1}{m}\left\|u_{m}\right\|_{\mathcal{L}_{-1}^{2}\left(\Omega_{e}\right)}^{2} .
$$

From the previous compact imbedding result, there is a subsequence, still denoted by $\left(u_{m}\right)_{m \in \mathbb{N}}$, which converges in $\mathcal{L}_{-1}^{2}\left(\Omega_{e}\right)$. Moreover, $(3.7)$ implies that $\left(\nabla u_{m}\right)_{m \in \mathbb{N}}$ converges to 0 in $L^{2}\left(\Omega_{e}\right)$. So, the sequence $\left(u_{m}\right)_{m \in \mathbb{N}}$ converges to a constant in $\mathcal{W}^{1,-1}\left(\Omega_{e}\right)$. We deduce again from relation $(3.7)$ that the trace of $u_{m}$ converge to 0 , thus the sequence $\left(u_{m}\right)_{m \in \mathbb{N}}$ converges to 0 , which is a contradiction with the normalization assumption.

TheOREM 3.1. Let $\alpha>0$, let $f$ be in the dual space $\left(\mathcal{W}^{1,-1}\left(\Omega_{e}\right)\right)^{*}$, and let $g \in$ $H^{-1 / 2}(\partial \Omega)$. Then, the following Laplace equation with Robin boundary condition

$$
\left\{\begin{array}{lc}
-\triangle u=f & \text { in } \Omega_{e} \\
u-\alpha \partial_{\nu} u=g & \text { on } \partial \Omega
\end{array}\right.
$$

admits a unique weak solution in $\mathcal{W}^{1,-1}\left(\Omega_{e}\right)$.

Proof. A variational formulation to (3.8) is given by

$$
\begin{aligned}
& \forall v \in \mathcal{W}^{1,-1}\left(\Omega_{e}\right), \\
& \int_{\Omega_{e}} \nabla u \nabla v d x+\frac{1}{\alpha} \int_{\partial \Omega} u v d \sigma=\int_{\Omega_{e}} f v d x+\frac{1}{\alpha} \int_{\partial \Omega} g v d \sigma
\end{aligned}
$$

It follows from the trace theorem that the left-hand side is a bounded bilinear form and the right -hand side is a bounded linear form. We study separately the $2 \mathrm{~d}$ and $3 \mathrm{~d}$ cases for the coercivity of the bilinear form.

i) If $d=2$, the coercivity is a direct consequence of lemma 3.2.

ii) If $d=3$, we deduce from Theorem 2.5.13 in [19], that there exists a constant $c>0$ such that $\forall u \in \mathcal{W}^{1,-1}\left(\Omega_{e}\right)$,

$$
\|u\|_{\mathcal{W}^{1,-1}\left(\Omega_{e}\right)} \leq c\|\nabla u\|_{L^{2}\left(\Omega_{e}\right)}
$$

Then, the left-hand side bilinear form in (3.9) is coercive.Thus, the result follows from the Lax-Milgram Theorem.

From the previous theorem, and without considering the asymptotic behavior $u(x)=O\left(\frac{1}{|x|^{d-1}}\right)$ as $|x| \rightarrow \infty$, we next construct solutions to (2.1) up to a constant. In a second step in order to establish the uniqueness of the solution, we will give necessary conditions on the trace of the interior and exterior solutions $\left.u\right|_{\partial \Omega-}$ and $\left.u\right|_{\partial \Omega+}$ respectively, such that the exterior solution satisfies the desired asymptotic behavior.

In the first step we split the equation (2.1) into two parts: the interior part

$$
\left\{\begin{array}{lr}
\triangle u_{i}=J_{s} & \text { in } \Omega \\
\frac{\partial u_{i}}{\partial \nu}=0 & \text { on } \partial \Omega \\
\int_{\partial \Omega} u_{i} d \sigma & =0
\end{array}\right.
$$


and the exterior part

$$
\left\{\begin{array}{lr}
\operatorname{div}\left[\left(1+(k(\omega)-1) \chi_{D}\right) \nabla u\right]=0 & \text { in } \Omega_{e}, \\
u=\xi \partial_{\nu} u+u_{i}+c_{u} & \text { on } \partial \Omega, \\
|u|=O\left(\frac{1}{|x|^{d-1}}\right) & \text { as }|x| \rightarrow \infty
\end{array}\right.
$$

where $c_{u}$ is a constant depending on $u$ that has to be fixed. It is clear that there exists a unique solution $u_{i}$ to (3.11). For a given constant $c_{u}$ theorem (3.1) guarantee the existence and uniqueness of solution to $(3.12)$ in $\mathcal{W}^{1,-1}\left(\Omega_{e}\right)$. Next, we show that the decay $O\left(\frac{1}{|x|^{d-1}}\right)$ of the exterior solution as $|x| \rightarrow+\infty$ will uniquely determine the constant $c_{u}$.

Lemma 3.3. Assume that $c_{u}$ is given. Then, the decay $O\left(\frac{1}{|x|^{d-1}}\right)$ at infinity implies

$$
\frac{1}{|\partial \Omega|} \int_{\partial \Omega} u d \sigma=c_{u}
$$

Proof. Recall the variational formulation for (3.8) with $f=0$ and $g=u_{i}+c_{u}$.

$$
\begin{aligned}
& \forall v \in \mathcal{W}^{1,-1}\left(\Omega_{e}\right) \\
& \int_{\Omega_{e}} \nabla u \nabla v d x+\frac{1}{\alpha} \int_{\partial \Omega}\left(u-c_{u}\right) v d \sigma=\frac{1}{\alpha} \int_{\partial \Omega} u_{i} v d \sigma .
\end{aligned}
$$

We distinguish two different cases:

i) $d=2$.

Since the constants belong to $\mathcal{W}^{1,-1}\left(\Omega_{e}\right)$, by taking $v=1$ in the previous variational formulation we obtain the desired result.

ii) $d=3$.

Let $B_{R}$ be a ball centered at 0 with radius $R>0$, and assume that $R$ is large enough such that $\bar{\Omega} \subset B_{R}$. Multiplying (3.12) by 1 and integrating by parts lead to

$$
\frac{1}{\alpha} \int_{\partial \Omega}\left(u-c_{u}\right) d \sigma=R^{2} \int_{S^{2}} \partial_{\nu} u(R t) d t
$$

where $S^{2}$ is the unit sphere in $\mathbb{R}^{3}$. Since $u$ harmonic in $B_{R}^{c}$, and decays as $O\left(\frac{1}{|x|^{2}}\right)$ when $|x| \rightarrow+\infty$, by expanding it in the spherical harmonic basis [19], we can easily obtain that $\partial_{\nu} u(R t)=O\left(\frac{1}{R^{3}}\right)$ as $R \rightarrow+\infty$ uniformly in $t \in S^{2}$. Consequently, the right hand term in the previous inequality tends to zero as $R \rightarrow+\infty$, which achieves the proof of the lemma.

Regarding the results of lemma 3.3, we can rewrite the problem (2.1) as follows

$$
\left\{\begin{array}{lr}
\operatorname{div}\left[\left(1+(k(\omega)-1) \chi_{D}\right) \nabla u\right]=0 & \text { in } \Omega_{e}, \\
u-\frac{1}{|\partial \Omega|} \int_{\partial \Omega} u d \sigma-\xi \partial_{\nu} u=u_{i} & \text { on } \partial \Omega, \\
|u|=O\left(\frac{1}{|x|^{d-1}}\right) & \text { as }|x| \rightarrow+\infty,
\end{array}\right.
$$

where $u_{i}$ is the unique solution to the system (3.11).

Let

$(3.15) \mathcal{W}_{\diamond}^{1,-1}\left(\Omega_{e}\right)=\left\{u \in \mathcal{W}^{1,-1}\left(\Omega_{e}\right) ;|u|=O\left(\frac{1}{|x|^{d-1}}\right)\right.$ as $\left.|x| \rightarrow+\infty\right\}$. 
We now state the main result of this subsection.

THEOREM 3.2. The forward problem (2.1) has a unique solution in $\mathcal{W}_{\diamond}^{1,-1}\left(\Omega_{e}\right)$.

Proof. Multiplying the equation (3.14) by $v$ in $\mathcal{W}_{\diamond}^{1,-1}\left(\Omega_{e}\right)$ and integrating by parts we obtain the following variational formulation

$$
\begin{aligned}
& \forall v \in \mathcal{W}^{1,-1}\left(\Omega_{e}\right) \\
& \int_{\Omega_{e}} \nabla u \nabla v d x+\frac{1}{\alpha} \int_{\partial \Omega}\left(u-f_{\partial \Omega} u\right)\left(v-f_{\partial \Omega} v\right) d \sigma=\frac{1}{\alpha} \int_{\partial \Omega} u_{i} v d \sigma,
\end{aligned}
$$

where $f_{\partial \Omega} u d \sigma=\frac{1}{|\partial \Omega|} \int_{\partial \Omega} u d \sigma$.

We claim that the left-hand bilinear form is coercive. In fact, when $d=3$ we deduce from [19], that the term $\int_{\Omega_{e}} \nabla u \nabla v d x$ is coercive in $\mathcal{W}^{1,-1}\left(\Omega_{e}\right)$. When $d=2$ it is proved in [12] that the term $\int_{\Omega_{e}} \nabla u \nabla v d x$ is also coercive in $\mathcal{W}_{\diamond}^{1,-1}\left(\Omega_{e}\right)$.

Then, by Lax-Milgram Theorem we obtain the desired result.

We introduce the fundamental solution $\Gamma$ to the Laplace operator in $\mathbb{R}^{d}$.

Definition 3.2. Denoting by $\omega_{d}$ the area of the unit sphere in $\mathbb{R}^{d}$, the fundamental solution to the Laplace operator is given by

$$
\Gamma(x, y)= \begin{cases}\frac{1}{2 \pi} \log (|x-y|) & d=2, \\ -\frac{1}{\omega_{d}}|x-y|^{2-d} & d \geq 3 .\end{cases}
$$

A direct consequence of theorem 3.1 is the following corollary.

Corollary 3.1. Let $y \in \Omega_{e}$ be fixed. Then, there exists a unique solution to the system

$$
\left\{\begin{array}{lc}
\triangle G(x, y)=\delta_{y} & \text { in } \Omega_{e}, \\
G-\xi \frac{\partial G}{\partial \nu}=\Gamma-\xi \frac{\partial \Gamma}{\partial \nu} & \text { on } \partial \Omega, \\
G-\Gamma \in \mathcal{W}^{1,-1}\left(\Omega_{e}\right) . &
\end{array}\right.
$$

The singular function $G(x, y)$ is the Green function of the Laplace operator in $\Omega_{e}$ with the Robin boundary condition.

\section{The Poincaré Variational Problem}

In this section, we introduce the Poincaré variational problem by following the approach in $[\mathbf{9}],[\mathbf{1 2}]$. We denote by $\mathcal{W}_{\diamond}^{1,-1}\left(\Omega_{e}\right)$, the subspace of functions $v \in$ $\mathcal{W}^{1,-1}\left(\Omega_{e}\right)$ satisfying $|v(x)|=O\left(\frac{1}{|x|^{d-1}}\right)$ as $|x| \rightarrow \infty$. It follows from [19], [12] that the space $\mathcal{W}_{\diamond}^{1,-1}\left(\Omega_{e}\right)$ endowed with the scalar product

$$
\langle u, v\rangle_{\mathcal{W}_{\diamond}^{1,-1}\left(\Omega_{e}\right)}:=\int_{\Omega_{e}} \nabla u \nabla v d x
$$

is a Hilbert space. Thus, the following bilinear form defines also an equivalent scalar product on $\mathcal{W}_{\diamond}^{1,-1}\left(\Omega_{e}\right)$,

$$
a(u, v):=\int_{\Omega_{e}} \nabla u \nabla v d x+\frac{1}{\xi} \int_{\partial \Omega} \bar{u} \bar{v} d \sigma
$$

where $\bar{u}:=u-\frac{1}{|\partial \Omega|} \int_{\partial \Omega} u d \sigma$. 
For $u \in \mathcal{W}_{\diamond}^{1,-1}\left(\Omega_{e}\right)$, we infer from the Riesz Theorem that there exist a unique $T u \in \mathcal{W}_{\diamond}^{1,-1}\left(\Omega_{e}\right)$ such that for all $v \in \mathcal{W}_{\diamond}^{1,-1}\left(\Omega_{e}\right)$,

$$
\int_{\Omega_{e}} \nabla T u \nabla v d x+\frac{1}{\xi} \int_{\partial \Omega} \overline{T u} \bar{v} d \sigma=\int_{D} \nabla u \nabla v d x .
$$

It is easy to check that the operator $T: \mathcal{W}_{\diamond}^{1,-1}\left(\Omega_{e}\right) \rightarrow \mathcal{W}_{\diamond}^{1,-1}\left(\Omega_{e}\right)$ is self-adjoint and bounded. The spectral problem for $T$ reads as: Find $(w, \lambda) \in \mathcal{W}_{\diamond}^{1,-1}\left(\Omega_{e}\right) \times \mathbb{R}$, $w \neq 0$, such that $\forall v \in \mathcal{W}_{\diamond}^{1,-1}\left(\Omega_{e}\right)$,

$$
\lambda \int_{\Omega_{e}} \nabla w \nabla v d x+\frac{\lambda}{\bar{\xi}} \int_{\partial \Omega} \bar{w} \bar{v} d \sigma=\int_{D} \nabla w \nabla v d x .
$$

Integrating by parts, we obtain that an eigenfunction $w$ is harmonic in $D$ and in $D^{\prime}:=\Omega_{e} \backslash \bar{D}$. We have the transmission and boundary conditions on $\partial D$

$$
\left.w\right|_{\partial D} ^{+}=\left.w\right|_{\partial D} ^{-},\left.\quad \partial_{\nu} w\right|_{\partial D} ^{+}=\left.\left(1-\frac{1}{\lambda}\right) \partial_{\nu} w\right|_{\partial D} ^{-},\left.\quad w\right|_{\partial \Omega}-\left.\xi \partial_{\nu} w\right|_{\partial \Omega}=\frac{1}{|\partial \Omega|} \int_{\partial \Omega} w d \sigma,
$$

where $\left.w\right|_{\partial D} ^{ \pm}(x)=\lim _{t \rightarrow 0} w(x+t \pm \nu(x))$ for $x \in \partial D$. In other words, $w$ is a solution to $(2.1)$ for $k=1-\frac{1}{\lambda}<0$, and $J_{s}=0$.

We define the space $\mathfrak{H}_{\diamond}$ as the space of harmonic functions in $D$ and in $D^{\prime}$ which are continuous across $\partial D$, satisfy a Robin boundary condition $\bar{u}=\xi \partial_{\nu} u$ on $\partial \Omega$ and the asymptotic behavior $|u|=O\left(\frac{1}{|x|^{d-1}}\right)$ as $|x| \rightarrow \infty$, and with a finite energy semi-norm

$$
\|u\|_{\mathfrak{H}_{\diamond}}^{2}:=\int_{\Omega_{e}}|\nabla u|^{2} d x+\frac{1}{\xi} \int_{\partial \Omega}|\bar{u}|^{2} d x .
$$

We remark that $T \mathfrak{H}_{\diamond} \subset \mathfrak{H}_{\diamond}$, thus $T$ defines a bounded operator from $\mathfrak{H}_{\diamond}$ into $\mathfrak{H}_{\diamond}$.

We define now, the single layer potential $S_{D}: H^{-1 / 2}(\partial D) \rightarrow \mathfrak{H}_{\diamond}$ and the Neumann-Poincaré operator $K_{D}^{*}: H^{-1 / 2}(\partial D) \rightarrow H^{-1 / 2}(\partial D)$ by

DeFinition 4.1. Let $\phi \in H^{-1 / 2}(\partial D)$, we define, for $x \in \Omega_{e}$

$$
S_{D}[\phi](x)=\int_{\partial D} G(x, y) \phi(y) d \sigma(y),
$$

and, for $x \in \partial D$,

$$
K_{D}^{*}[\phi](x)=\int_{\partial D} \frac{\partial}{\partial \nu_{x}} G(x, y) \phi(y) d \sigma(y) .
$$

Since $D$ is smooth $K_{D}^{*}$ is compact on $H^{-1 / 2}(\partial D)$ (see lemma 2.13 in [8]). Since the function $G$ and the Newtonian potential $\Gamma$ have equivalent weak singularities as $x \rightarrow y$ (see Lemma 2.14 in [7] for a sketch of the proof and section 2.5.5 in [19] for the regularity), the single layer potential $S_{D}$ satisfies the Plemjel jump relations across the boundary $\partial D$, that is,

$$
\partial_{\nu} S_{D}[\phi]_{ \pm}(x)=\left( \pm \frac{1}{2} I+K_{D}^{*}\right)[\phi](x) .
$$

We next characterize the spectrum of $T$.

Theorem 4.1. The Poincaré variational operator $T$ has the following decomposition

$$
T=\frac{1}{2} I+K
$$


where $K$ is a compact, self-adjoint operator. Let $w_{n}^{ \pm}, n \geq 1$ be the eigenfunctions of $T$ associated to the eigenvalues $\left(\lambda_{n}^{ \pm}\right)_{n \geq 1}$, we have the following min-max principle

$$
\begin{aligned}
\lambda_{1}^{-} & =\min _{0 \neq w \in \mathfrak{H}_{\diamond}} \frac{\int_{D}|\nabla w(x)|^{2} d x}{\int_{\Omega_{e}}|\nabla w(x)|^{2} d x+\frac{1}{\xi} \int_{\partial \Omega}|\bar{w}|^{2} d x} \\
\lambda_{n}^{-} & =\min _{0 \neq w \in \mathfrak{H}_{\diamond}, w \perp w_{1}^{-}, \cdots, w_{n-1}^{-}} \frac{\int_{D}|\nabla w(x)|^{2} d x}{\int_{\Omega_{e}}|\nabla w(x)|^{2} d x+\frac{1}{\xi} \int_{\partial \Omega}|\bar{w}|^{2} d x} \\
& =\min _{F_{n} \subset \mathfrak{H}_{\diamond}, \operatorname{dim}\left(F_{n}\right)=n} \max _{w \in F_{n}} \frac{\int_{D}|\nabla w(x)|^{2} d x}{\int_{\Omega_{e}}|\nabla w(x)|^{2} d x+\frac{1}{\xi} \int_{\partial \Omega}|\bar{w}|^{2} d x},
\end{aligned}
$$

and similarly

$$
\begin{aligned}
\lambda_{1}^{+} & =\max _{0 \neq w \in \mathfrak{H}_{\diamond}} \frac{\int_{D}|\nabla w(x)|^{2} d x}{\int_{\Omega_{e}}|\nabla w(x)|^{2} d x+\frac{1}{\xi} \int_{\partial \Omega}|\bar{w}|^{2} d \sigma} \\
\lambda_{n}^{+} & =\max _{0 \neq w \in \mathfrak{H}_{\diamond}, w \perp w_{1}^{+}, \cdots, w_{n-1}^{+}} \frac{\int_{D}|\nabla w(x)|^{2} d x}{\int_{\Omega_{e}}|\nabla w(x)|^{2} d x+\frac{1}{\xi} \int_{\partial \Omega}|\bar{w}|^{2} d x} \\
& =\max _{F_{n} \subset \mathfrak{H}_{\diamond}, \operatorname{dim}\left(F_{n}\right)=n} \min _{w \in F_{n}} \frac{\int_{D}|\nabla w(x)|^{2} d x}{\int_{\Omega_{e}}|\nabla w(x)|^{2} d x+\frac{1}{\xi} \int_{\partial \Omega}|\bar{w}|^{2} d x} .
\end{aligned}
$$

Proof. We define the operator $R: \mathfrak{H}_{\diamond} \rightarrow \mathfrak{H}_{\diamond}$, for all $v \in \mathfrak{H}_{\diamond}$,

$$
2\left(\int_{\Omega_{e}} \nabla R u \nabla v d x+\frac{1}{\xi} \int_{\partial \Omega} \overline{R u} \bar{v} d \sigma\right)=\int_{D} \nabla u \nabla v d x-\int_{D^{\prime}} \nabla u \nabla v d x-\frac{1}{\xi} \int_{\partial \Omega} \bar{u} \bar{v} d \sigma .
$$

We observe that $K$ is bounded and self-adjoint. A direct calculation shows that

$$
T=\frac{1}{2} I+R
$$

It is shown in [8] that the single layer potential $S_{D}: H^{-1 / 2}(\partial D) \rightarrow H^{1 / 2}(\partial D)$ is invertible in dimension three, and we can modify slightly $S_{D}$ to show the invertibility in dimension two.

Integrating by parts the right-hand side of (4.11), and using the jump relation (4.7), we find

$$
\int_{\Omega_{e}} \nabla R u \nabla v d x+\frac{1}{\xi} \int_{\partial \Omega} \overline{R u} \bar{v} d \sigma=\int_{\partial D} K_{D}^{*}\left[S_{D}^{-1}\left[\left.u\right|_{\partial D}\right]\right] v d \sigma .
$$

Since $K_{D}^{*}$ is compact the operator $R$ is compact.

From Fredholm's alternative, $T$ is a Fredholm operator of index 0 , and the spectral decomposition (4.9), (4.10) follows the min-max principle.

Corollary 4.1. Let $u \in \mathfrak{H}_{\diamond}$, then $u$ has the spectral decomposition.

$$
u(x)=\sum_{n=1}^{\infty} u_{n}^{ \pm} w_{n}^{ \pm}(x),
$$

where

$$
u_{n}^{ \pm}=\int_{\Omega_{e}} \nabla u \nabla w_{n}^{ \pm} d x+\frac{1}{\xi} \int_{\partial \Omega} \bar{u} \bar{w}_{n}^{ \pm} d x
$$




\section{Spectral decomposition of the solution $u(x, \omega)$}

TheOREm 5.1. Let $u(x, \omega)$ be the unique solution to the system (2.1). Then, the following decomposition holds:

$$
\begin{aligned}
u(x, \omega) & =u_{0}(x)+u_{f}(x, k(\omega)) \\
& =u_{0}(x)+\frac{1}{\xi} \sum_{n=1}^{\infty} \frac{\int_{\partial \Omega} u_{i} w_{n}^{ \pm} d \sigma}{1+\lambda_{n}^{ \pm}(k(\omega)-1)} w_{n}^{ \pm}(x), \quad x \in \Omega_{e},
\end{aligned}
$$

where $u_{0} \in \mathcal{W}_{\diamond}^{1,-1}\left(\Omega_{e}\right)$ is the unique solution to the system:

$$
\left\{\begin{array}{lr}
\triangle v=0 & \text { in } \Omega_{e} \backslash \bar{D} \\
\nabla v=0 & \text { in } D \\
v-f_{\partial \Omega} v d \sigma=\xi \partial_{\nu} v+u_{i} & \text { on } \partial \Omega \\
|v|=O\left(\frac{1}{|x|^{d-1}}\right) & \text { as }|x| \rightarrow \infty
\end{array}\right.
$$

and $u_{i} \in L^{2}(\Omega)$ is the unique solution to the equation:

$$
\left\{\begin{array}{l}
\triangle v=J_{s} \quad \text { in } \Omega, \\
\partial_{\nu} v=0 \quad \text { on } \partial \Omega, \\
\int_{\partial \Omega} v d \sigma=0 .
\end{array}\right.
$$

Proof. Let $u_{\star}$ be the unique solution to

$$
\left\{\begin{array}{lr}
\triangle v=J_{s} & \text { in } \Omega, \\
\left.\partial_{\nu} v\right|_{-}=0 & \text { on } \partial \Omega, \\
\left.\int_{\partial \Omega} v\right|_{-} d \sigma=0, & \text { in } \Omega_{e} \\
\triangle v=0 & \text { on } \partial \Omega \\
\left.v\right|_{+}-\left.f_{\partial \Omega} v\right|_{+} d \sigma-\left.v\right|_{-}=\left.\xi \partial_{\nu} v\right|_{+} & \text {as }|x| \rightarrow \infty . \\
|v|=O\left(\frac{1}{|x|^{d-1}}\right) & \rightarrow \infty
\end{array}\right.
$$

Denote $\mathfrak{u}:=u-u_{\star}, \mathfrak{u}$ is therefore harmonic in $D$ and in $\Omega_{e} \backslash \bar{D}$. Moreover, it satisfies

$$
\left.\mathfrak{u}\right|_{+}-\left.f_{\partial \Omega} \mathfrak{u}\right|_{+} d \sigma=\left.\xi \partial_{\nu} \mathfrak{u}\right|_{+} \text {on } \partial \Omega
$$

Then $\mathfrak{u} \in \mathfrak{H}_{\diamond}$, and admits the following spectral decomposition:

$$
\mathfrak{u}(x)=\sum_{n=1}^{\infty} \mathfrak{u}_{n}^{ \pm} w_{n}^{ \pm}(x),
$$

where

$$
\mathfrak{u}_{n}^{ \pm}=\int_{\Omega_{e}} \nabla \mathfrak{u} \nabla w_{n}^{ \pm} d x+\frac{1}{\xi} \int_{\partial \Omega} \overline{\mathfrak{u}} \bar{w}_{n}^{ \pm} d \sigma
$$

By definition, $\mathfrak{u}$ is the unique solution to

$$
\left\{\begin{array}{lr}
-\operatorname{div}(\gamma(x, \omega) \nabla \mathfrak{u})=\operatorname{div}\left(\gamma(x, \omega) \nabla u_{\star}\right) & \text { in } \Omega_{e}, \\
\mathfrak{u}-\xi \partial_{\nu} \mathfrak{u}=\frac{1}{|\partial \Omega|} \int_{\partial \Omega} \mathfrak{u} d \sigma & \text { on } \partial \Omega \\
|\mathfrak{u}|=O\left(\frac{1}{|x|^{d-1}}\right) & \text { as }|x| \rightarrow \infty
\end{array}\right.
$$

Multiplying the first equation in (5.7) by $w_{n}^{ \pm}$, and integrating by parts over $\Omega_{e}$, we have

$$
\mathfrak{u}_{n}^{ \pm}=\frac{\int_{\Omega_{e}} \operatorname{div}\left(\gamma(x, \omega) \nabla u_{\star}\right) w_{n}^{ \pm} d x}{1+\lambda_{n}^{ \pm}(k(\omega)-1)}
$$


The term $\int_{\Omega_{e}} \operatorname{div}\left(\gamma(x, \omega) \nabla u_{\star}\right) w_{n}^{ \pm} d x$ can be understood as a dual product between $\operatorname{div}\left(\gamma(x, \omega) \nabla u_{\star}\right) \in\left(\mathcal{W}^{1,-1}\left(\Omega_{e}\right)\right)^{*}$ and $w_{n}^{ \pm} \in \mathcal{W}^{1,-1}\left(\Omega_{e}\right)$. It can be simplified by means of integration by parts:

$$
\begin{aligned}
& \int_{\Omega_{e}} \operatorname{div}\left(\gamma(x, \omega) \nabla u_{\star}\right) w_{n}^{ \pm} d x \\
& =-\int_{\Omega_{e}} \gamma(x, \omega) \nabla u_{\star} \nabla w_{n}^{ \pm} d x-\int_{\partial \Omega} \partial_{\nu} u_{\star} w_{n}^{ \pm} d \sigma \\
& =-\int_{\Omega_{e}} \nabla u_{\star} \nabla w_{n}^{ \pm} d x-(k(\omega)-1) \int_{D} \nabla u_{\star} \nabla w_{n}^{ \pm} d x-\frac{1}{\xi} \int_{\partial \Omega}\left(\left.u_{\star}\right|_{+}-\left.u_{\star}\right|_{-}\right) w_{n}^{ \pm} d \sigma \\
& =-\int_{\Omega_{e}} \nabla u_{\star} \nabla w_{n}^{ \pm} d x-(k(\omega)-1) \int_{D} \nabla u_{\star} \nabla w_{n}^{ \pm} d x-\frac{1}{\xi} \int_{\partial \Omega}\left(\overline{\left.u_{\star}\right|_{+}}-\overline{\left.u_{\star}\right|_{-}}\right) \bar{w}_{n}^{ \pm} d \sigma \\
& =-\left[\frac{1}{\lambda_{n}^{ \pm}}+(k(\omega)-1)\right] \int_{D} \nabla u_{\star} \nabla w_{n}^{ \pm} d x+\frac{1}{\xi} \int_{\partial \Omega} \overline{\left.u_{\star}\right|_{-}} w_{n}^{ \pm} d \sigma,
\end{aligned}
$$

where $\overline{\left.u_{\star}\right|_{-}}$is exactly the unique solution to (7.5), it means, $\overline{\left.u_{\star}\right|_{-}}=u_{i}$. Thus, it follows that

$$
\mathfrak{u}_{n}^{ \pm}=-\frac{\int_{D} \nabla u_{\star} \nabla w_{n}^{ \pm} d x}{\lambda_{n}^{ \pm}}+\frac{\int_{\partial \Omega} u_{i} w_{n}^{ \pm} d \sigma}{\xi\left[1+\lambda_{n}^{ \pm}(k(\omega)-1)\right]} .
$$

Let $\tilde{u_{0}} \in \mathfrak{H}_{\diamond}$ be the unique solution to the system

$$
\left\{\begin{array}{lr}
\triangle \tilde{u_{0}}=0 & \text { in } \Omega_{e} \backslash \bar{D}, \\
\nabla \tilde{u_{0}}=\nabla u_{\star} & \text { in } D, \\
\tilde{u_{0}}-\xi \frac{\partial \tilde{u_{0}}}{\partial \nu}=\frac{1}{|\partial \Omega|} \int_{\partial \Omega} \tilde{u_{0}} d \sigma & \text { on } \partial \Omega, \\
\left|\tilde{u_{0}}\right|=O\left(\frac{1}{|x|^{d-1}}\right) & \text { as }|x| \rightarrow \infty .
\end{array}\right.
$$

Since $w_{n}^{ \pm}$is an eigenfunction of $T$ and $\tilde{u_{0}} \in \mathfrak{H}_{\diamond}$, we have

$$
\int_{D} \nabla u_{\star} \nabla w_{n}^{ \pm} d x=\lambda_{n}^{ \pm}\left[\int_{\Omega_{e}} \nabla \tilde{u_{0}} \nabla w_{n}^{ \pm} d x+\frac{1}{\xi} \int_{\partial \Omega} \overline{u_{0}} \bar{w}_{n}^{ \pm} d \sigma\right]
$$

which gives

$$
\mathfrak{u}_{n}^{ \pm}=-\left[\int_{\Omega_{e}} \nabla \tilde{u_{0}} \nabla w_{n}^{ \pm} d x+\frac{1}{\xi} \int_{\partial \Omega} \tilde{\tilde{u_{0}}} \bar{w}_{n}^{ \pm} d \sigma\right]+\frac{\int_{\partial \Omega} u_{i} w_{n}^{ \pm} d \sigma}{\xi\left[1+\lambda_{n}^{ \pm}(k(\omega)-1)\right]} .
$$

On the other hand a simple calculations yields

$$
u_{\star}-\tilde{u}_{0}=u_{0} .
$$

Combining (5.13) amd (5.14), the decomposition (5.1) follows.

Corollary 5.1. Let $x \in \partial \Omega$, the function $k \mapsto u_{f}(x, k)$ is meromorphic in $\mathbb{C}$. The poles of $u_{f}(x, k)$ are the real solutions to the equations

$$
1+\lambda_{n}^{ \pm}(k-1)=0, \quad n \geq 1
$$

where $\lambda_{n}^{ \pm}$are the eigenvalues of the Poincaré variational operator $T$.

The poles of $u_{f}(x, k)$ in the previous corollary are given by $k_{n}^{ \pm}=\left(1-\frac{1}{\lambda_{n}^{ \pm}}\right) \in \mathbb{R}_{-}$, and they can be ordered as follows:

$$
k_{1}^{-} \leq k_{2}^{-} \leq \cdots<-1<\cdots \leq k_{2}^{+} \leq k_{1}^{+}<0
$$


We remark that -1 is the only accumulation point of the sequence of poles, it means $k_{n}^{ \pm}$tends to -1 as $n \rightarrow \infty$.

\section{Uniqueness and stability estimates}

By modifying slightly the proofs in sections 3 and 4 of [9], we establish the uniqueness of the inverse weakly electric fish, and the following stability estimate

Theorem 6.1. Let $D$ and $\widetilde{D}$ be two inclusions in $\mathfrak{D}$. Denote by $u$ and $\tilde{u}$ respectively the solution of (2.1) with the inclusion $D$ (resp. $\widetilde{D})$. Let

$$
\varepsilon=\sup _{x \in \partial \Omega, \omega \in[\underline{\omega}, \bar{\omega}]}|u-\tilde{u}| .
$$

Then, there exist constants $C$ and $\tau \in(0,1)$, such that the following estimate holds:

$$
|D \triangle \widetilde{D}| \leq C\left(\frac{1}{\ln \left(\varepsilon^{-1}\right)}\right)^{\tau} .
$$

Here, $\triangle$ denotes the symmetric difference and the constants $C$ and $\tau$ depend only on $J_{s}, \Omega, \mathfrak{D}$ and $\Sigma=\{k(\omega) ; \omega \in(\underline{\omega}, \bar{\omega})\}$.

Moreover, if the boundaries are analytic, we have a Hölder-type stability estimate.

Theorem 6.2. Assume that $d=2$, and let $D$ and $\widetilde{D}$ be two analytic inclusions in $\mathfrak{D}$. Denote by $u$ and $\tilde{u}$ respectively the solution of (2.1) with the inclusion $D$ (resp. $\widetilde{D})$. Let

$$
\varepsilon=\sup _{x \in \partial \Omega, \omega \in[\underline{\omega}, \bar{\omega}]}|u-\tilde{u}| .
$$

Then, there exist constants $C$ and $\tau^{\prime} \in(0,1)$, such that the following estimate holds:

$$
|D \triangle \widetilde{D}| \leq C \varepsilon^{\tau^{\prime}} \text {. }
$$

Here, $\triangle$ denotes the symmetric difference and the constants $C$ and $\tau$ depend only on $J_{s}, \Omega, \mathfrak{D}$ and $\Sigma$.

These results show that the reconstruction of $D$ is improving according to the regularity of its boundary. Precisely, the stability estimates vary from logarithmic to Hölder. They can also be extended to a larger class of as non-star shaped and non-connected domains. In this paper for the sake of simplicity we do not handle such general cases.

The proofs of Theorems 6.1 and 6.2 follow exactly the arguments presented in sections 3-4 of [9], and we do not reproduce them here. They fundamentally rely on the spectral decompositions of $u$ and $\tilde{u}$. Thus, concerning stability estimates, one of the main contributions of our paper is to show that such spectral decompositions also hold for the solutions to exterior problems, and therefore yield similar estimates. In the proof, the electric potential on the electric fish skin is split into two parts $u(x, \omega)=u_{0}(x)+u_{f}(x, k(\omega))$, and separates between $k$-dependent and $k$-independent parts. In fact $u_{0}$ corresponds to the response of the same inclusion filled with a perfect conductor, that is $u_{0}$ is the limit of $u$ when $k$ tends to infinity. Since $k \rightarrow u$ is meromorphic and its poles are simple, $\left.u_{f}(x, k)\right|_{\partial \Omega}$ can be recovered using unique continuation for meromorphic functions. $\left.\left(u_{0}, \partial_{\nu} u_{0}\right)\right|_{\partial \Omega}$ can in turn be retrieved from $\left.u(., \omega)\right|_{\partial \Omega}, \omega \in(\underline{\omega}, \bar{\omega})$. The problem of determining $D$ from the Cauchy data of $u_{0}$ has been studied by several authors (see for instance $[\mathbf{1 3}, \mathbf{2}]$ and references therein). 


\section{Numerical identification of the target fish}

In this section, we develop a numerical scheme to determine the position and shape of the target fish. Regarding the decay of the solution of (2.1) as $|x| \rightarrow+\infty$, we first reduce the computational domain by taking a Dirichlet boundary condition on a large disk $B$ containing the fish $\Omega$ and the inclusion $D$. In fact we will show that a spectral decomposition similar to (5.1) holds in the truncated problem. Using this spectral decomposition, our numerical algorithm splits into two main steps. In the first step we retrieve the frequency depend part $u_{f}$, and in the second step we reconstruct the inclusion from the Cauchy data of $u_{0}$ on $\partial \Omega$ obtained in the first step. Precisely, we recover the fish target by using an optimization scheme that minimizes a given energy functional on the boundary $\partial \Omega$ with respect to the shape of the inclusion.

7.1. The mathematical model in a truncated domain. In order to implement the numerical identification method, we need to reduce the system (2.1) to a bounded domain. Let $B$ be a centered disk large enough such that it contains the electric fish $\Omega$ and the target fish $D$. We substitute (2.1) by the system

$$
\left\{\begin{array}{lr}
\triangle \tilde{u}=J_{s} & \text { in } \Omega, \\
\operatorname{div}[\gamma(x, \omega) \nabla \tilde{u}]=0 & \text { in } B \backslash \bar{\Omega} \\
\left.\partial_{\nu} \tilde{u}\right|_{-}=0 & \text { on } \partial \Omega, \\
\left.\tilde{u}\right|_{+}-\left.\tilde{u}\right|_{-}=\left.\xi \partial_{\nu} \tilde{u}\right|_{+} & \text {on } \partial \Omega, \\
\tilde{u}=0 & \text { on } \partial B \\
\int_{\partial B} \partial_{\nu} \tilde{u} d \sigma=0 . &
\end{array}\right.
$$

The standard theory of elliptic partial differential equations shows that (7.1) admits a unique solution in

$$
W_{0}:=\left\{u \in H^{1}(B \backslash \bar{\Omega}) \cup H^{1}(\Omega), u=0 \text { on } \partial B, \int_{\partial B} \partial_{\nu} \tilde{u} d \sigma=0\right\} .
$$

We introduce the equivalent scalar product on $W_{0}$ and the associated Poincaré variational operator $\tilde{T}$.

$$
\tilde{a}(u, v):=\int_{B \backslash \bar{\Omega}} \nabla u \nabla v d x+\frac{1}{\xi} \int_{\partial \Omega} \bar{u} \bar{v} d \sigma .
$$

For $u \in \mathfrak{H}$, by the Riez theorem, there exists aunique $\widetilde{T} u \in W_{0}$ such that for all $v \in W_{0}$,

$$
\int_{B \backslash \bar{\Omega}} \nabla \widetilde{T} u \nabla v d x+\frac{1}{\xi} \int_{\partial \Omega} \widetilde{T} u v d \sigma=\int_{D} \nabla u \nabla v d x
$$

We introduce also the space $\mathfrak{H}_{\diamond} \subset W_{0}$ of the functions which are harmonic in $D$ and in $B \backslash \overline{(\Omega \cup D)}$, and which satisfy the zero Robin boundary condition $u-\xi \partial_{\nu} u=0$ on $\partial \Omega$.

Then, $\widetilde{T}$ has similar spectral elements denoted $\left(w_{n}^{ \pm}, \tilde{\lambda}_{n}^{ \pm}\right)$as $T$. We follow the analysis in the unbounded case to derive a similar spectral decomposition for $\tilde{u}$. 
TheOREM 7.1. Let $\tilde{u}(x, \omega)$ be the unique solution to the system (7.1). Then the following decomposition holds, for $x \in B \backslash \bar{\Omega}$,

$$
\begin{aligned}
& \tilde{u}(x, \omega)=\tilde{u}_{0}(x)+u_{f}(x, k(\omega)) \\
& =\tilde{u}_{0}(x)+\frac{1}{\xi} \sum_{n=1}^{\infty} \frac{\int_{\partial \Omega} \tilde{u}_{i} \tilde{w}_{n}^{ \pm} d \sigma}{1+\tilde{\lambda}_{n}^{ \pm}(k(\omega)-1)} \tilde{w}_{n}^{ \pm}(x),
\end{aligned}
$$

where $u_{0} \in W_{0}$ is the unique solution to the system:

$$
\left\{\begin{array}{lr}
\triangle v=0 & \text { in } B \backslash \overline{(\Omega \cup D),} \\
\nabla v=0 & \text { in } D, \\
v-f_{\partial \Omega} v d \sigma=\xi \partial_{\nu} v+u_{i} & \text { on } \partial \Omega, \\
v=0 & \text { on } \partial B, \\
\int_{\partial B} \partial_{\nu} v d \sigma=0, &
\end{array}\right.
$$

and $u_{i} \in L^{2}(\Omega)$ is the unique solution to the equation:

$$
\left\{\begin{array}{l}
\triangle v=J_{s} \quad \text { in } \Omega, \\
\partial_{\nu} v=0 \quad \text { on } \partial \Omega, \\
\int_{\partial \Omega} v d \sigma=0 .
\end{array}\right.
$$

7.2. Retrieval of the frequency independent part. We consider $M$ frequencies of measurements $\omega_{1}, \omega_{2}, \cdots, \omega_{M}$. Since $1 / 2$ is the unique accumulation point of the eigenvalues $\left(\tilde{\lambda}_{n}^{ \pm}\right)_{n \in \mathbb{N}}$, we only consider the $N_{f}$ first eigenvalues as unknowns, and we approximate the others eigenvalues by $1 / 2$. In fact it has been shown in [17] that if $D$ is $C^{\beta}$ with $\beta \geq 2$ then for any $\alpha>-2 \beta+3$, we have

$$
\left|\tilde{\lambda}_{n}^{ \pm}-1 / 2\right|=o\left(n^{\alpha}\right), \quad n \rightarrow+\infty .
$$

If the boundary of $D$ is $C^{\infty}$ smooth, then the eigenvalues will decay faster than any power. Recently H. Kang and his collaborators have shown the exponential convergence of the eigenvalues in the case of analytic inclusions [11]. Therefore, we make the following approximation, for $x \in \Omega, 1 \leq p \leq M$,

$$
\tilde{u}\left(x, \omega_{p}\right) \approx \tilde{u}_{0}(x)+\sum_{n=1}^{N_{f}} \frac{v_{n}^{ \pm}(x)}{1+\tilde{\lambda}_{n}^{ \pm}\left(k\left(\omega_{p}\right)-1\right)}+\frac{2}{k\left(\omega_{p}\right)+1} v_{N_{f}+1}(x),
$$

where

and

$$
v_{n}^{ \pm}(x)=\frac{1}{\xi} \int_{\partial \Omega} \tilde{u}_{i} \tilde{w}_{n}^{ \pm} d \sigma \tilde{w}_{n}^{ \pm}(x)
$$

$$
v_{N_{f}+1}(x)=\frac{1}{\xi} \sum_{n>N_{f}} \int_{\partial \Omega} \tilde{u}_{i} \tilde{w}_{n}^{ \pm} d \sigma \tilde{w}_{n}^{ \pm}(x) .
$$

By a simple integration by parts, we have, for all $n \in \mathbb{N}$,

$$
\frac{1}{\xi} \int_{\partial \Omega} \tilde{u}_{i} \tilde{w}_{n}^{ \pm} d \sigma=\int_{B \backslash \bar{\Omega}} \nabla \tilde{u}_{\star} \nabla \tilde{w}_{n}^{ \pm} d x+\frac{1}{\xi} \int_{\partial \Omega} \tilde{u}_{\star} \tilde{w}_{n}^{ \pm} d \sigma
$$

where $\tilde{u}_{\star}$ is the unique solution in $W_{0}$ to

$$
\left\{\begin{array}{lr}
\triangle \tilde{u}_{\star}=0 & \text { in } B \backslash \bar{\Omega}, \\
\tilde{u}_{\star}-f \tilde{u}_{\star} d \sigma-\xi \partial_{\nu} \tilde{u}_{\star}=\tilde{u}_{i} & \text { on } \partial \Omega, \\
\tilde{u}_{\star}=0 & \text { on } \partial B \\
\int_{\partial B} \partial_{\nu} \tilde{u}_{\star} d \sigma=0 . &
\end{array}\right.
$$


In other words, the function $\sum_{n=1}^{\infty} v_{n}^{ \pm}$is the orthogonal projection of the function $\tilde{u}_{\star}$ on the space $\tilde{H}_{\diamond}$.

On the other hand $\tilde{u}_{0}$ satisfies, for all $n \in \mathbb{N}, n \geq 1$

$$
\begin{aligned}
& \int_{B \backslash \bar{\Omega}} \nabla \tilde{u}_{0} \nabla \tilde{w}_{n}^{ \pm} d x+\frac{1}{\xi} \int_{\partial \Omega} \tilde{u}_{0} \tilde{w}_{n}^{ \pm} d \sigma \\
& =\int_{B \backslash \overline{(\Omega \cup D)}} \nabla \tilde{u}_{0} \nabla \tilde{w}_{n}^{ \pm} d x+\frac{1}{\xi} \int_{\partial \Omega} \tilde{u}_{0} \tilde{w}_{n}^{ \pm} d \sigma \\
& =-\int_{\partial \Omega} \tilde{u}_{0} \partial_{\nu} \tilde{w}_{n}^{ \pm} d \sigma+\frac{1}{\xi} \int_{\partial \Omega} \hat{u}_{0} \tilde{w}_{n}^{ \pm} d \sigma-\int_{\partial D} \tilde{u}_{0} \partial_{\nu} \tilde{w}_{n}^{ \pm} d \sigma \\
& =-\tilde{u}_{0} \int_{\partial D} \partial_{\nu} \tilde{w}_{n}^{ \pm} d \sigma=0 .
\end{aligned}
$$

As $\tilde{u}_{\star}-\tilde{u}_{0} \in \mathfrak{H}_{\diamond}$, the orthogonal projection of $\tilde{u}_{\star}$ on the space $\tilde{H}_{\diamond}$ is $\tilde{u}_{\star}-\tilde{u}_{0}$.

Then, the formula (7.6) becomes

$$
\begin{aligned}
\tilde{u}\left(x, \omega_{p}\right) \approx & \frac{k\left(\omega_{p}\right)-1}{k\left(\omega_{p}\right)+1} \tilde{u}_{0}(x)+\frac{2}{k\left(\omega_{p}\right)+1} \tilde{u}_{\star}(x) \\
& +\sum_{n=1}^{N_{f}}\left(\frac{1}{1+\tilde{\lambda}_{n}^{ \pm}\left(k\left(\omega_{p}\right)-1\right)}-\frac{2}{k\left(\omega_{p}\right)+1}\right) v_{n}^{ \pm}(x) .
\end{aligned}
$$

The previous equation can be formulated with the following matrix form.

For $x \in \partial \Omega$, we define the vectors

$$
\begin{gathered}
\tilde{U}\left(x, \omega_{1}, \ldots, \omega_{M}\right)=\left(\tilde{\mathfrak{u}}\left(x, \omega_{j}\right)\right) \\
V(x)=\left(\tilde{u}_{0}(x), v_{1}^{+}(x), v_{1}^{-}(x), \ldots, v_{N_{f}}^{+}(x), v_{N_{f}}^{-}(x)\right),
\end{gathered}
$$

and

$$
\begin{gathered}
L\left(\widetilde{\lambda_{1}^{ \pm}}, \ldots, \widetilde{\lambda_{N_{f}}^{ \pm}}, \omega_{1}, \ldots \omega_{M}\right)=\left(L_{i}\right)_{1 \leq i \leq M}, \text { where } \\
\left.\left.L_{i}(x)=\left(q_{0}\left(\omega_{i}\right), q\left(\widetilde{\lambda_{1}^{+}}, \omega_{i}\right), q\left(\widetilde{\lambda_{1}^{-}}, \omega_{i}\right), \cdots, q \widetilde{{\lambda_{N_{f}}^{+}}_{1}}, \omega_{i}\right), q \widetilde{{\overline{\lambda_{N_{f}}^{-}}}_{1}} \omega_{i}\right)\right) .
\end{gathered}
$$

Here

$$
\begin{array}{r}
\tilde{\mathfrak{u}}(x, \omega)=\tilde{u}(x, \omega)-\frac{2}{k(\omega)+1} \tilde{u}_{\star}(x), \\
q_{0}(\omega)=\frac{k(\omega)-1}{(k(\omega)+1)}, \quad \text { and } q(\tilde{\lambda}, \omega)=\frac{1}{1+\widetilde{\lambda}(k(\omega)-1)}-\frac{2}{k(\omega)+1} .
\end{array}
$$

The matrix formulation of (7.10) becomes then

$$
\tilde{U}^{T}\left(x, \omega_{1}, \omega_{2}, \cdots, \omega_{M}\right) \approx L\left(\widetilde{\lambda_{1}^{ \pm}}, \ldots, \widetilde{\lambda_{N_{f}}^{ \pm}}, \omega_{1}, \ldots \omega_{M}\right) V^{T}(x)
$$

So, the vector $V$ can be obtained by the formula,

$$
V^{T}(x) \approx\left(L^{T} L\right)^{\dagger} L^{T} \tilde{U}^{T}\left(x, \omega_{1}, \cdots \omega_{M}\right) .
$$


where $\left(L^{T} L\right)^{\dagger}$ is the pseudo-inverse of the matrix $L^{T} L$. The conditioning of the matrix $\left(L^{T} L\right)^{\dagger}$ depends on the distance between the frequency sampling values $\omega_{0}$. The approximate $\tilde{u}_{0}(x)$ is then given by the first coefficient of the vector $V(x)$.

7.3. Identification of the target fish. In this section, we consider a numerical scheme to identify the inclusion $D \in \mathfrak{D}$ from the knowledge of $\left.\tilde{u}_{0}\right|_{\partial \Omega}$ recovered in the previous section. The scheme is based on minimizing the functional

$$
J(D)=\frac{1}{2} \int_{\partial \Omega} \sum_{i=1}^{P}\left|\tilde{u}_{0}-\tilde{u}_{m e a s}^{(i)}\right|^{2} d \sigma+\varepsilon_{T} \int_{\partial D} d \sigma
$$

where $\tilde{u}$ is the solution to (7.4). $P$ designs the total number of measurements, we take here $P=10$. For $1 \leq i \leq 10$, we use the fact that the electric fish can swim around the target, $\tilde{u}_{\text {meas }}^{(i)}$ is the measured Dirichlet data corresponding to the case when the electric fish is located at the $i$-th position. These quantities are obtained in the previous step by retrieving the frequency dependent part from the multifrequency measurements. The term $\varepsilon_{T} \int_{\partial D} d \sigma$ represents the Tikhonov regularization.

7.3.1. Shape derivative. Let $D_{\varepsilon}$ be the perturbed domain, given by

$$
\partial D_{\varepsilon}=\{\tilde{x}: \tilde{x}=x+\varepsilon h(x) \nu(x), x \in \partial D\},
$$

where $h \in \mathcal{C}^{1}(\partial D)$ and $\nu$ denote the unit outward normal vector.

THEOREM 7.2. We denote by $\tilde{u}_{0}$ and by $\tilde{u}_{0, \varepsilon}$ respectively the solutions to the equation (7.4) with the inclusion $D$ (resp. $D_{\varepsilon}$ ). Then, the following relation holds, for $x \in \partial \Omega$,

$$
\tilde{u}_{0, \varepsilon}(x)=\tilde{u}_{0}(x)+\varepsilon \tilde{u}_{h}(x)+o(\varepsilon),
$$

where $\tilde{u}_{h}$ is the solution to the following equation

$$
\left\{\begin{array}{lr}
\triangle v=0 & \text { in } B \backslash \overline{(\Omega \cup D),} \\
\nabla v=0 & \text { in } D, \\
v=-\left.h \partial_{\nu} \tilde{u}_{0}\right|_{+} & \text {on } \partial D, \\
v-f v d \sigma-\xi \partial_{\nu} v=0 & \text { on } \partial \Omega, \\
v=0 & \text { on } \partial B, \\
\int_{\partial B} \partial_{\nu} v d \sigma=0, &
\end{array}\right.
$$

Proof. The result can be proved using layer potential techniques by following the proof of Theorem 3.1 in [10].

7.3.2. Gradient descent algorithms. We assume that our domain $D$ is star shaped, centered at the origin and that its boundary $\partial D$ can be described by the Fourier series:

$$
\partial D=\left\{r(\theta)\left(\begin{array}{c}
\cos \theta \\
\sin \theta
\end{array}\right) \mid \theta \in[0 ; 2 \pi)\right\}, r=\sum_{n=-N}^{N} c_{n} f_{n},
$$
where $C=\left(\begin{array}{c}c_{-N} \\ c_{-N+1} \\ \vdots \\ c_{N}\end{array}\right) \in \mathbb{R}^{2 N+1}, f_{n}(\theta)=\cos (n \theta)$ for $0 \leq n \leq N$ and $f_{n}(\theta)=$
$\sin (n \theta)$ for $-N \leq n<0$. 
Using (7.15) and integration by parts, we deduce the expressions of the shape derivatives corresponding to each Fourier coefficient, for $-N \leq n \leq N$,

$$
\frac{\partial J}{\partial c_{n}}=\int_{B \backslash \overline{\Omega \cup D}} \nabla w \nabla \tilde{u}_{h} d x+\varepsilon_{T} \int_{\partial D} \kappa h d \sigma,
$$

where $h(\theta)=f_{n}(\theta)\left\langle\left(\begin{array}{c}\cos \theta \\ \sin \theta\end{array}\right), \nu\right\rangle, \kappa$ represents the curvature of $\partial D$ and $w$ is the solution of the following equation

$$
\left\{\begin{array}{lr}
\triangle w=0 & \text { in } B \backslash \overline{\Omega \cup D} \\
\partial_{\nu} w=0 & \text { on } \partial D \\
\partial_{\nu} w=\tilde{u}-\tilde{u}_{\text {meas }} & \text { on } \partial \Omega \\
w=0 & \text { on } \partial B .
\end{array}\right.
$$

Now, we are ready to introduce our iterative algorithm:

(1) Calculate the interior solution $\tilde{u}_{i}$.

(2) Using (7.12), we retrieve the frequency independent part $\tilde{u}_{0}$ from the multifrequency measurements. We get the Dirichlet data $\left(\hat{u}_{\text {meas }}^{(i)}\right)_{1 \leq i \leq P}$.

(3) Chose an initial domain $D_{0}$.

(4) For each iteration, $j>0$ :

(a) Using (7.4) associated to the domain $D_{j}$ for which the boundary $\partial D_{j}$ is obtained from (7.16).

(b) Calculate the shape derivatives $\frac{\partial J}{\partial c_{n}}$ for all $-N \leq n \leq N$ by (7.17).

(c) Update the parameters of the domain $C_{j+1}=C_{j}-\alpha \nabla_{C} J\left(C_{j}\right)$ with $\alpha>0$.

(d) If the updated domain boundary touches $\partial \Omega$ or if $J\left(C_{j+1}\right)>J\left(C_{j}\right)$, reduce the size of $\alpha$.

(5) When $\left|\nabla J\left(C_{j}\right)\right|$ becomes smaller than a fixed threshold, we stop.

FIGURE 1. Interior solution to the equation (7.1) 


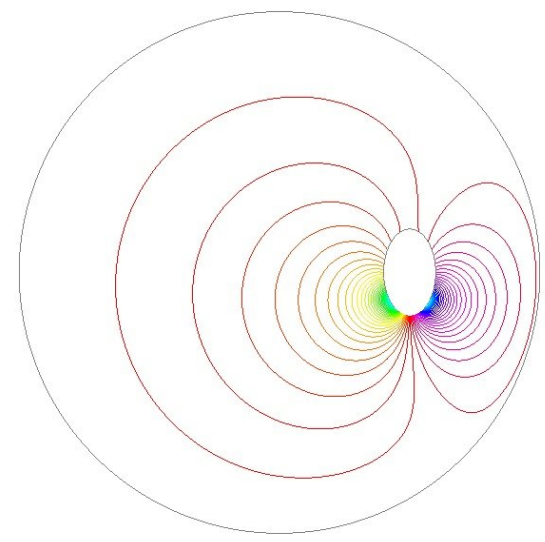

(A) without inclusion

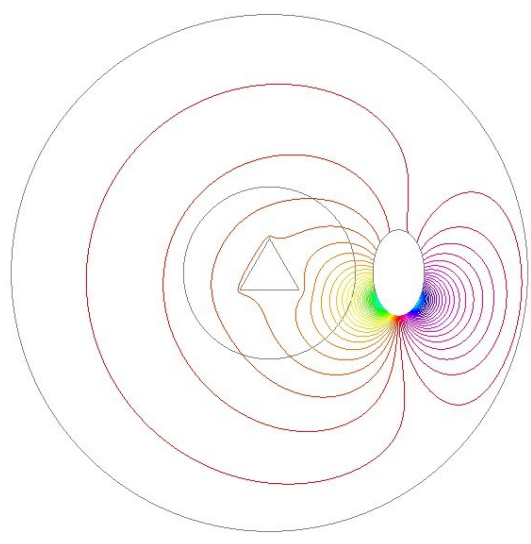

(B) inclusion presents

Figure 2. Solutions to (7.1) in the presence/absence of inclusion
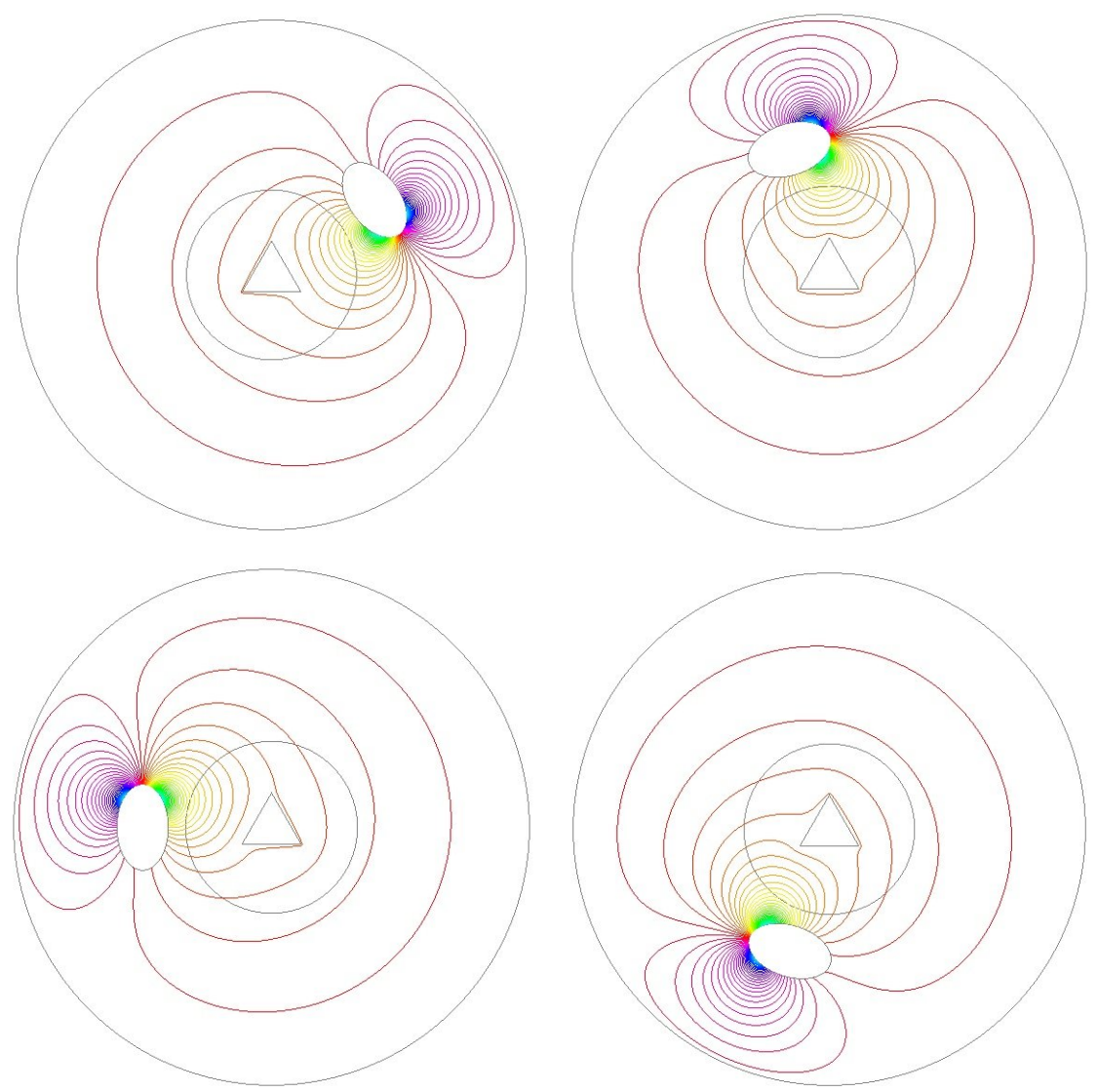

FiguRE 3. Different positions of the electric fish 
7.4. Numerical experiments. The setting of all numerical tests is as follows:

- We use FreeFem++ for our numerical experiments [15].

- $B$ is a centered ball with the radius $R_{B}=30$.

- $\Omega$ is an ellipse defined by the equation: $\frac{x^{2}}{3^{2}}+\frac{y^{2}}{4^{2}} \leq 1$.

- We assume that the source function is given by a dipole type source, i. e., in the formula (2.2), $M=2, \alpha_{1}=100, \alpha_{2}=-100, x_{s}^{(1)}=(-3,1)$, and $x_{s}^{(2)}=(-3,-1)$.

The interior solution is illustrated in Figure 1.

- We take advantage of the fact that the electric fish can swim around the target. We chose $P=10$ different locations to measure the multifrequency electric potentials, those 10 positions are equi-distributed on the circle with a radius 15 . Figure 3 shows the 4 locations of them.

- The multifrequency conductivity follows the model $k(\omega)=k_{r}+i n \omega_{0}$ with $k_{r}=5, \omega_{0}=0.5$ and $n$ are integers from 0 to $7[3]$.

- Only the first eigenvalues are taken into consideration, and their apriori estimations are settled as $\widetilde{\lambda_{1}^{+}}=\frac{3}{4}, \widetilde{\lambda_{1}^{-}}=\frac{1}{4}$ respectively in all cases.

- The initial estimation of domain $D$ : a centered disk with a radius $\frac{1}{2}$.

- We consider the first 9 Fourier coefficients: $N=9$.

- We set the Tikhonov regularization coefficient $\varepsilon_{T}=0.01$.

- We use P1 finite elements for the numerical resolution of the PDEs.

- At each iteration, we remesh the domain to adapt to the new predicted shape of the domain.

- The algorithm stops if $|\nabla J|<10^{-6}$ or if the number of iterations exceeds 100 .

We present here several numerical simulations of the algorithm. We first present in Table (1), errors in the reconstruction method of $\tilde{u}_{0}$. Here, errors are the $L^{2}$-norm of the difference $\tilde{u}_{0 \text { reconstruct }}-\tilde{u}_{0}$ :

$$
\operatorname{error}\left(\tilde{u}_{0 \text { reconstruct }}\right):=\sqrt{\int_{\partial \Omega}\left|\tilde{u}_{0 \text { reconstruct }}-\tilde{u}_{0}\right|^{2} d \sigma} .
$$

We show in the following figures the targets and the reconstruction result. We calculate also the relative symmetric difference $\left|D_{i} \triangle D_{\text {target }}\right| /\left|D_{\text {target }}\right|$ during the iterations, and we plot the curves of the symmetric difference in terms of iteration number. We finally give the relative symmetric difference of each case in Table 2.

\section{Conclusion}

Using the mathematical model of the weakly electric fish introduced by Ammari and al. in [3], we addressed the question of existence and uniqueness of the solution to the direct problem. We have established the uniqueness and stability estimates of the fish target recovery problem using a single measurement, under the assumption that its conductivity profile is known. We provided a gradient descent algorithm to determine the shape and location of the target.

\section{Acknowledgments}

The authors were partially supported by the AGIR-HOMONIM grant from Université Grenoble-Alpes, and by the Labex PERSYVAL-Lab (ANR-11-LABX0025-01). 


\begin{tabular}{|c|c|c|c|c|}
\hline$\bullet$ & ellipse & triangle & star & displaced disk \\
\hline measure 1 & 0.10132 & 0.06892 & 0.01411 & 0.05330 \\
\hline measure 2 & 0.07466 & 0.07384 & 0.03364 & 0.05010 \\
\hline measure 3 & 0.01637 & 0.05302 & 0.07036 & 0.04712 \\
\hline measure 4 & 0.00910 & 0.04580 & 0.05242 & 0.04388 \\
\hline measure 5 & 0.03460 & 0.06548 & 0.02822 & 0.03792 \\
\hline measure 6 & 0.06959 & 0.08078 & 0.03558 & 0.03408 \\
\hline measure 7 & 0.05795 & 0.06175 & 0.02743 & 0.03585 \\
\hline measure 8 & 0.02060 & 0.03240 & 0.03819 & 0.03891 \\
\hline measure 9 & 0.00675 & 0.02760 & 0.07660 & 0.04054 \\
\hline measure 10 & 0.03074 & 0.03623 & 0.05292 & 0.04663 \\
\hline
\end{tabular}

TABLE 1. Errors between $\tilde{u}_{0 r e c o n s t r u c t}$ and $\tilde{u}_{0}$

\begin{tabular}{|c|c|c|c|c|}
\hline$\bullet$ & ellipse & triangle & star & displaced disk \\
\hline$\left|D_{i} \triangle D_{\text {target }}\right| /\left|D_{\text {target }}\right|$ & 0.07128 & 0,1988 & 0.4232 & 0.16805 \\
\hline
\end{tabular}

TABLE 2. Relative symmetric difference

\section{References}

[1] Robert A Adams. Compact sobolev imbeddings for unbounded domains with discrete boundaries. Journal of Mathematical Analysis and Applications, 24(2):326 - 333, 1968.

[2] Giovanni Alessandrini, Elena Beretta, Edi Rosset, and Sergio Vessella. Optimal stability for inverse elliptic boundary value problems with unknown boundaries. Ann. Sc. Norm. Super. Pisa, Cl. Sci., IV. Ser., 29(4):755-806, 2000.

[3] Habib Ammari, Thomas Boulier, and Josselin Garnier. Modeling active electrolocation in weakly electric fish. SIAM Journal on Imaging Sciences, 6(1):285-321, 2013.

[4] Habib Ammari, Thomas Boulier, Josselin Garnier, Wenjia Jing, Hyeonbae Kang, and Han Wang. Target identification using dictionary matching of generalized polarization tensors. Foundations of Computational Mathematics, 14(1):27-62, 2014.

[5] Habib Ammari, Thomas Boulier, Josselin Garnier, and Han Wang. Shape recognition and classification in electro-sensing. Proceedings of the National Academy of Sciences, 111(32):11652-11657, 2014.

[6] Habib Ammari, Josselin Garnier, Laure Giovangigli, Wenjia Jing, and Jin-Keun Seo. Spectroscopic imaging of a dilute cell suspension. Journal de Mathématiques Pures et Appliquées, 105(5):603 - 661, 2016.

[7] Habib Ammari and Hyeonbae Kang. Reconstruction of small inhomogeneities from boundary measurements. Number 1846. Springer Science \& Business Media, 2004.

[8] Habib Ammari and Hyeonbae Kang. Polarization and moment tensors: with applications to inverse problems and effective medium theory, volume 162. Springer Science \& Business Media, 2007.

[9] Habib Ammari and Faouzi Triki. Identification of an inclusion in multifrequency electric impedance tomography. Communications in Partial Differential Equations, 42(1):159-177, 2017.

[10] Habib Ammari, Faouzi Triki, and Chun-Hsiang Tsou. Numerical determination of anomalies in multifrequency electrical impedance tomography. arXiv preprint arXiv:1704.04878, 2017.

[11] Kazunori Ando, Hyeonbae Kang, and Yoshihisa Miyanishi. Exponential decay estimates of the eigenvalues for the neumann-poincar 'e operator on analytic boundaries in two dimensions. arXiv preprint arXiv:1606.01483, 2016. 


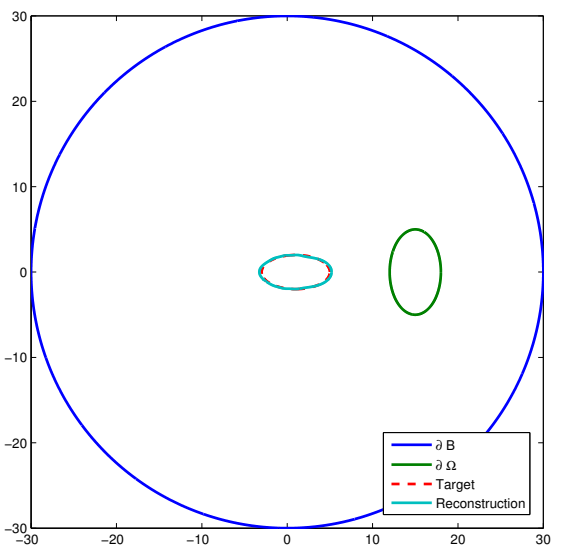

(A) Overall view

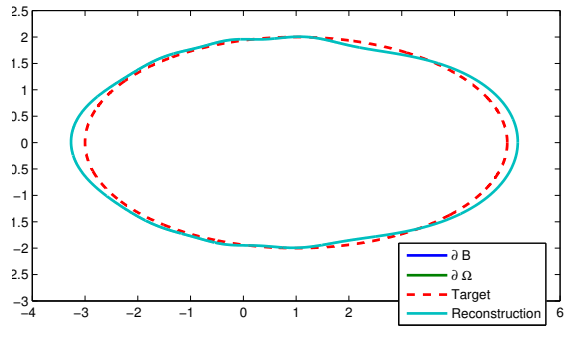

(B) Near the target

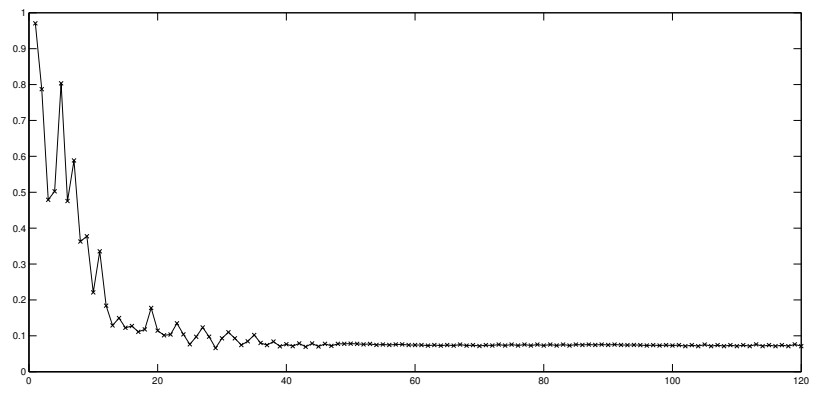

(C) Evolution of relative symmetric differences

Figure 4. Reconstruction of an ellipse

[12] Eric Bonnetier and Faouzi Triki. On the spectrum of the poincaré variational problem for two close-to-touching inclusions in 2d. Archive for Rational Mechanics and Analysis, 209(2):541$567,2013$.

[13] AL Bukhgeim, J Cheng, and M Yamamoto. Conditional stability in an inverse problem of determining a non-smooth boundary. Journal of mathematical analysis and applications, 242(1):57-74, 2000.

[14] Oscar M Curet, Neelesh A Patankar, George V Lauder, and Malcolm A MacIver. Aquatic manoeuvering with counter-propagating waves: a novel locomotive strategy. Journal of The Royal Society Interface, 8(60):1041-1050, 2011.

[15] F. Hecht. New development in freefem++. J. Numer. Math., 20(3-4):251-265, 2012.

[16] Vincent Lebastard, Christine Chevallereau, Ali Amrouche, Brahim Jawad, Alexis Girin, Frédéric Boyer, and Pol Bernard Gossiaux. Underwater robot navigation around a sphere using electrolocation sense and kalman filter. In Intelligent Robots and Systems (IROS), 2010 IEEE/RSJ International Conference on, pages 4225-4230. IEEE, 2010.

[17] Yoshihisa Miyanishi and Takashi Suzuki. Eigenvalues and eigenfunctions of double layer potentials. Transactions of the American Mathematical Society, 2017.

[18] Peter Moller. Electric fishes: history and behavior, volume 17. Chapman \& Hall, 1995.

[19] Jean-Claude Nédélec. Acoustic and electromagnetic equations: integral representations for harmonic problems, volume 144. Springer Science \& Business Media, 2001. 


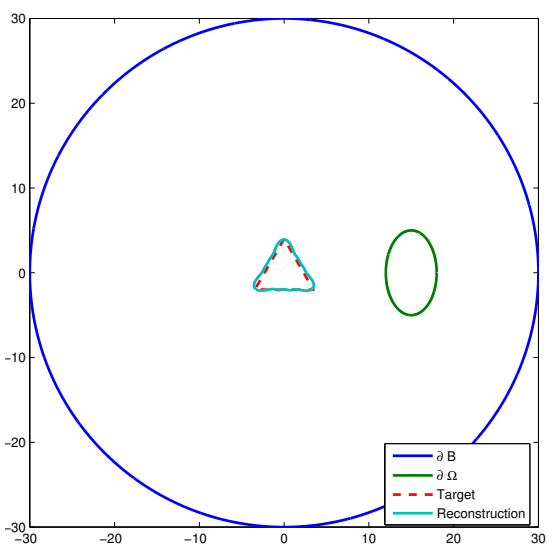

(A) Overall view

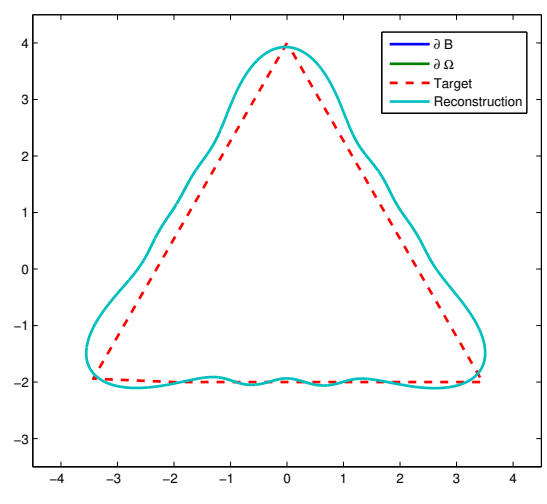

(B) Near the target

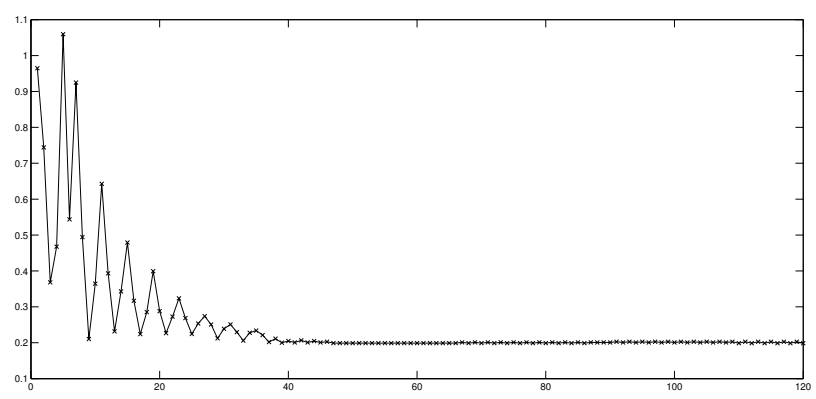

(c) Evolution of relative symmetric differences

FigURE 5. Reconstruction of a triangle

Institut Fourier, Université Grenoble-Alpes, BP 74, 38402 Saint-Martin-d?Hères Cedex, France

E-mail address: eric.bonnetier@univ-grenoble-alpes.fr

Laboratoire Jean Kuntzmann, UMR CNRS 5224, Université Grenoble-Alpes, 700 Avenue Centrale, 38401 Saint-Martin-D'Hères, France

E-mail address: faouzi.triki@univ-grenoble-alpes.fr

Laboratoire Jean Kuntzmann, UMR CNRS 5224, Université Grenoble-Alpes, 700 Avenue Centrale, 38401 Saint-Martin-D'Hères, France

E-mail address: chun-hsiang.tsou@univ-grenoble-alpes.fr 


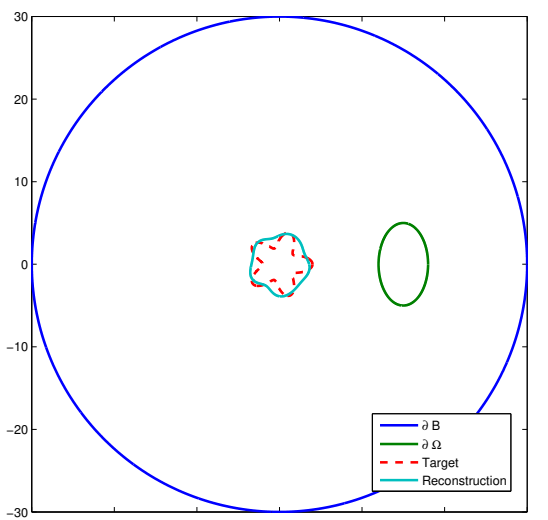

(A) Overall view

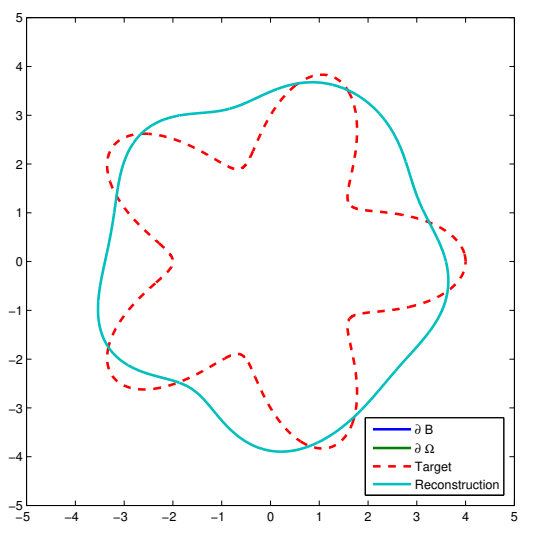

(B) Near the target

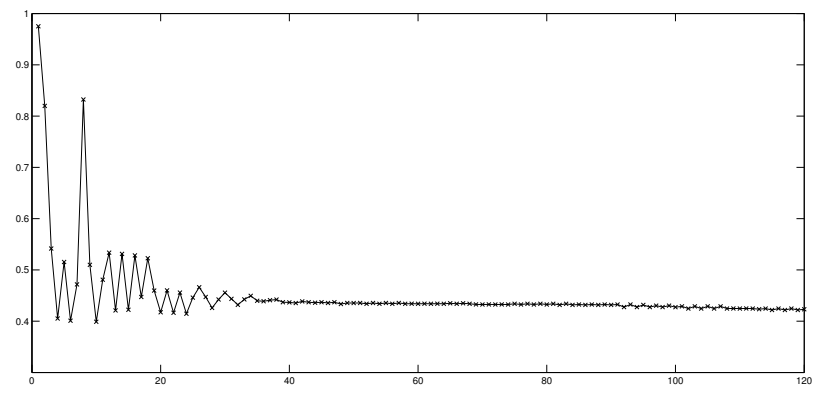

(C) Evolution relative of symmetric differences

Figure 6. Reconstruction of a star-shape domain 


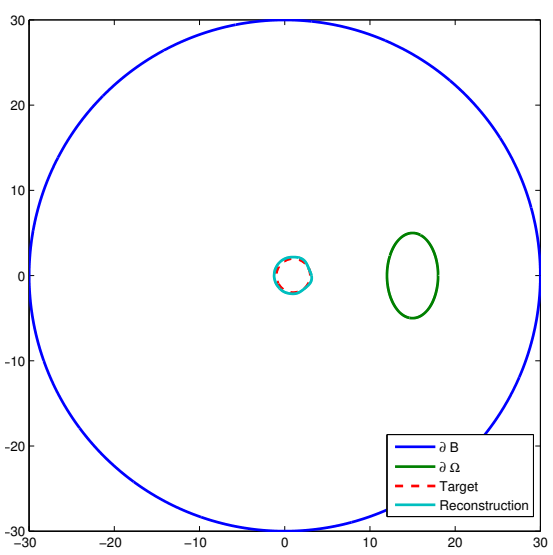

(A) Overall view

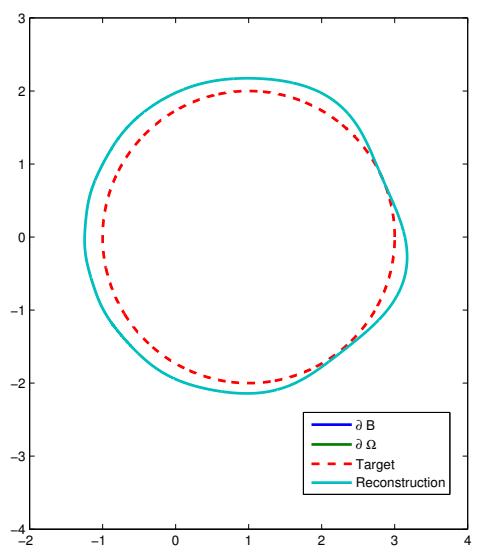

(B) Near the target

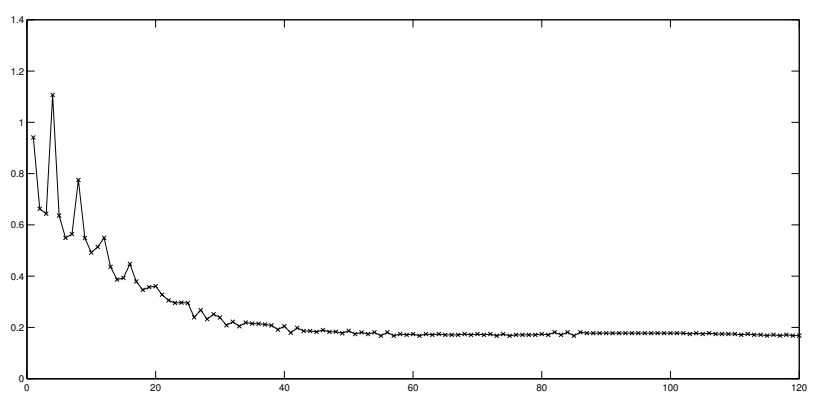

(C) Evolution of relative symmetric differences

FiguRE 7. Reconstruction of a displaced disk 